\title{
Adaptive Skins: Towards New Material Systems
}

\author{
Ofir Albag, Maria Anishchenko, Giulia Grassi and Ingrid Paoletti
}

\begin{abstract}
The aim of this chapter is to shed light on the potentialities of smart material systems in architectural skins. The research on new materials has triggered new solutions, based on emergent technologies, for envelope design. Adaptive facades, exploiting stimulus-responsive materials and intelligent control systems, are able to change in response to environmental conditions, thus enhancing users' comfort and improving energy savings. Two different case studies are here illustrated: the first is a dynamic shading system based on shape memory alloys; the second example concerns a soft-robotics weather-responsive skin. Both projects are described following the same workflow structure in order to highlight similarities and differences. Indeed, the sections are outlining the steps for design, simulation and fabrication of the case studies, and are divided as follows: description of the project, methodology and design strategy, simulation and prototyping.
\end{abstract}

Keywords Adaptive facades $\cdot$ Shape memory alloys $\cdot$ Soft robotics • Computational design $\cdot$ Smart materials

\section{The Role of Adaptivity}

In the last decade, the concept of adaptive facade has arisen from the debate on energy efficiency in buildings. Indeed, the term "adaptive" refers to a system which constantly changes as a result of climate variations. A plethora of studies showed that envelopes are responsible in large part for buildings' energy consumption. Dynamic skins are able to optimally balance energy demand and occupants' comfort. Multiperformance skins were introduced as a paradigm for an "all season walls", providing protection in all weather conditions.

O. Albag

Milan, Italy

M. Anishchenko · G. Grassi ( $₫)$ - I. Paoletti

Architecture, Built Environment and Construction Engineering-ABC Department, Politecnico di Milano, Milan, Italy

e-mail: giulia.grassi@polimi.it

B. Daniotti et al. (eds.), Digital Transformation of the Design, Construction and Management Processes of the Built Environment, Research for Development, https://doi.org/10.1007/978-3-030-33570-0_19 
The research on facades is currently focusing on the design of mechanisms that allow adaptation, especially exploiting material properties or intelligent sensing/actuating devices. Thus, the advent of smart materials has led to a new conception of design and manufacturing, where the process can be simulated and optimized by embedding material properties and programming behavioural patterns.

This chapter is an attempt to identify fundamental morphological characteristics of kinetic adaptive facades by illustrating two case studies that has been developed within this research line.

\section{Stimulus-Responsive Materials and Control Systems}

Envelopes are considered as a demarcation line between external and internal conditions, a safety boundary that protects us, as well as a threshold between energy field transition (high-energy to low energy or different state of energy). However, studies on new materials and kinetic mechanisms are eliciting a new vision of facades, as the space where the transition happens, an interactive multifaceted space. Therefore, the role of the designer becomes designing, thus programming, the interaction.

Stimuli-responsive materials (SRMs) are able to enact intelligent behaviours in response to an external stimulus. According to Ritter (2007) SRMs can be classified on the basis of the effect produced by the stimulus as: property changing, energy exchanging and matter exchanging. Stimuli can be identified also with environmental external factors that influence human comfort, to name a few: solar radiation, outdoor temperature, wind, humidity, precipitation, noise, and pollution. The first two can be seen as the major elements impacting the design of the system from a performative perspective. Moreover, temperature and light are also the two most impacting triggers for activating SRMs. The effective control of environmental factors is a crucial point for the successful operation of adaptive facades. Indeed, without proper detection tools and control systems, the envelopes become senseless. According to Addington and Schodek (2005), control systems can be divided in two categories: extrinsic (active) and intrinsic (passive) (Table 1).

Extrinsic control systems rely on automated regulation and consist of sensors, processors, actuators and control logic. They are indeed capable of detecting feedback, by comparing the current configuration with the desired one and, if necessary,

Table 1 List of properties of extrinsic and intrinsic control systems

\begin{tabular}{l|l}
\hline Extrinsic & Intrinsic \\
\hline Gradual & Immediate response-direct actuation \\
\hline Automated (sensors-processors-actuators) & Based on material properties \\
\hline Users feedback & No user interaction \\
\hline Energy intensive & Energy saving \\
\hline Constant maintenance & No reaction to unexpected events \\
\hline
\end{tabular}


adjusting the behaviour of the building envelope. Notable applications of extrinsic systems are Al-Bahr Towers, One Ocean Pavilion and Institute du Monde Arab.

Intrinsic (or passive) control systems are characterized by the inherent adaptive capacity of materials, or kinetic components, constituting the facade. The environmental stimulus is the trigger for such self-adjusting behaviour. However, there is no filter: environmental inputs are directly transformed into actions. Therefore, it is of fundamental importance to carefully study the material response to the stimuli and simulating/ testing the whole functioning prototype. Some examples exploiting intrinsic control systems include: SmartWrap at Cooper-Hewitt National Design Museum and HygroSkin Meteorosensitive Pavilion.

The hybrid combination of the two control systems enables to take full advantage of smart materials and electronics. The following sections will illustrate two case studies of adaptive facades, shading light on design strategies for programmable materials.

\section{Shape Memory Alloys for a Dynamic Shading System}

The aim of this project is to design a kinetic system with low-maintenance and high degree of flexibility for its application. Hence, the main objective is to investigate an intrinsic technology in which environmental inputs could be directly transformed into actions, without external decision-making component. Stimuli-responsive materials constitute the perfect candidate, thanks to their ability of changing their shape as a response to an external trigger.

Shape memory alloys (SMA) are materials with the ability to recover their original shape after deformation, at the presence of proper conditions (Huang et al. 2010). SMA enables to create simple modules which can react to temperature change and modify their shape through an immediate response. This makes them universal materials that can work simultaneously as sensors and actuators, and react without the need of a control system. The shape memory effect is achieved thanks to the solidstate phase change of SMA. Most of the existing materials have three phases: solid, liquid and gas and react on a change of temperature, depending on characteristics of the material. A broadly similar process happens in a solid-state phase change. The material is a solid both before and after the transformation because the molecules remain very close together throughout. SMAs flip back and forth between two solid crystalline states called austenite and martensite.

\subsection{Methodology and Design Strategy}

The workflow is divided into three consecutive phases. It starts with the analysis of the daylight performance and understanding the need of the dynamic solar shading. The second step is to define the tools and methodologies. The final step of the research 
is the design of the shading module, the simulations of its effectiveness and the fabrication of a physical prototype.

The project is situated in southern Siberia, in the Russian city of Tomsk. The temperature there can fluctuate from -40 in winter to +30 in summer. According to the local construction regulations, fixed shading is prohibited due to the cold climate in winter. Nevertheless, shading in summer is needed. Evidently dynamic shading can play an important role and permit different solutions.

The objective of the work is to create a solar screening system which is light and easily deployable, but at the same time resistant to weather conditions. Therefore, the principle of origami, Miura-Ori pattern (Fig. 1a), coupled together with SMA joints, provide a dynamic shell mechanism. This pattern permits to create a light and thin structure, which is at the same time stiff enough to sustain external forces (Schenk and Guest 2014). The Shape Memory Alloy hinges can be actuated without any control system and be completely autonomous. In order to add manual control to the system, the alloys can respond to electrical stimuli as they heat up and subsequently change shape (Fig. 1b).

A large-scale application of such deployable mechanism requires high flexibility, stiffness and lightness. One material technology that well suits these requirements is "Wood-skin". It is created of digitally fabricated wooden tiles, sandwiching a nylon and polymer mesh in between (Mok 2016). The benefit of this material that the SMA foil can be inserted in-between the layers of wood, creating an invisible hinge.

The scheme of hinges allocation for the Miura-Ori pattern is following the logic of the origami folds. All the folds are divided in positive $\left(+180^{\circ}\right)$ and negative ones $\left(-180^{\circ}\right)$, the so-called "mountain and valley folds". The SMA hinge is attached to the folding-sheet with micro-nuts inside the layers of plywood.

(a)

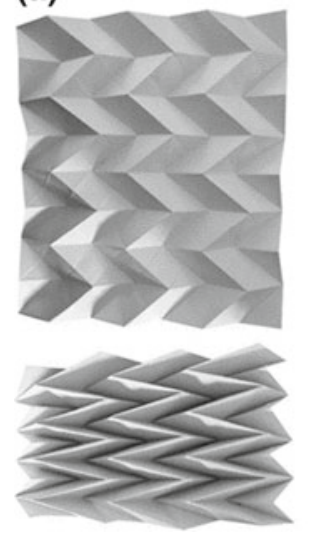

(b)

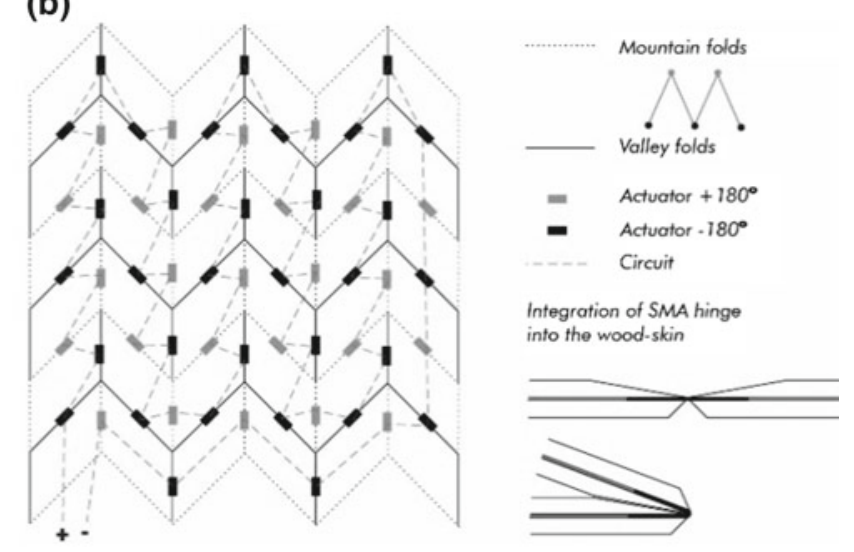

Fig. 1 a Miura-ori origami pattern. b Scheme of the allocation of the actuator 


\subsection{Simulation and Prototyping}

The study of the Shape Memory Alloy is supported with a series of experiments with nitinol foil. The shape setting of the foil is done with the help of a high temperature furnace and high-voltage infra-red lamps for testing. The model is realized in a scale of 1:10. The wood-skin material is modelled with pieces of plywood of $1 \mathrm{~mm}$ thickness attached to the non-stretch fabrics.

To set the shape of the Nitinol it should be heated up to a temperature of 400 $500{ }^{\circ} \mathrm{C}$ for $10-25 \mathrm{~min}$, depending on the material's composition and thickness, then immediately cooled down to room temperature. Rectangular pieces of Nitinol with size $10-12 \mathrm{~mm}$ with thickness of $0.1 \mathrm{~mm}$ are folded to $180^{\circ}$ and put on a ceramic base weighted by a $5 \mathrm{~kg}$ block of metal and left in the heated furnace on $400^{\circ}$ for $15 \mathrm{~min}$.

The pieces of preprogramed nitinol are then attached to the plywood with tape. The choice of tape is due to the small scale of the model. In the full-sized prototype, small bolts could be used instead to create a stiffer connection. Testing the smallscale prototype, the connections work well and react on the changes in temperature (Fig. 2a, b) (Anishchenko 2018).

The research results in the design of a shading device for a window (Fig. 3). A modular system can vary in size and be applied to new and existing buildings.

The technology's efficiency is simulated with Ladybug for Grasshopper. The simulation date is set to the July 1 . The surface of the window glass is divided in smaller areas and the level of solar radiation is analysed in the centre of each area.

(a)

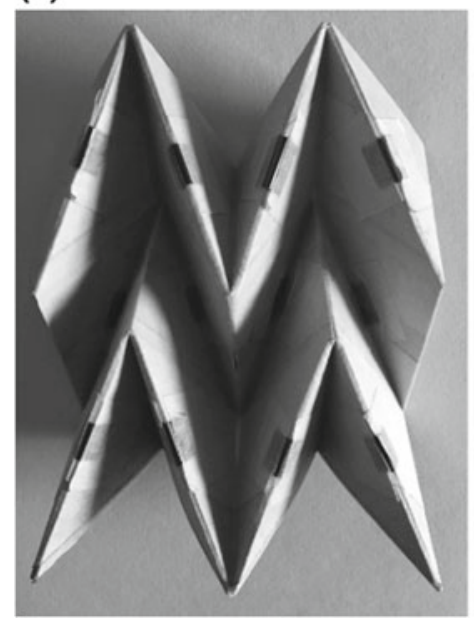

(b)

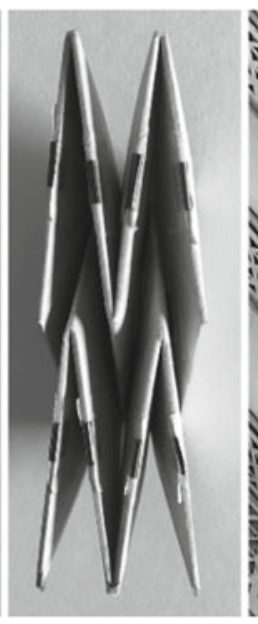

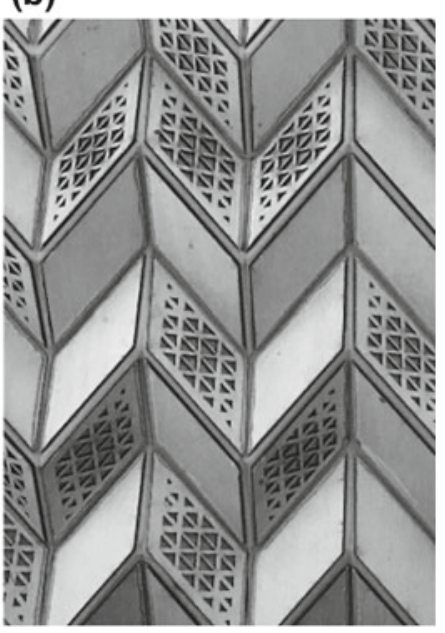

Fig. 2 a Physical prototype scale 1:10 with implemented SMA; b Physical prototype with cut out geometrical pattern 

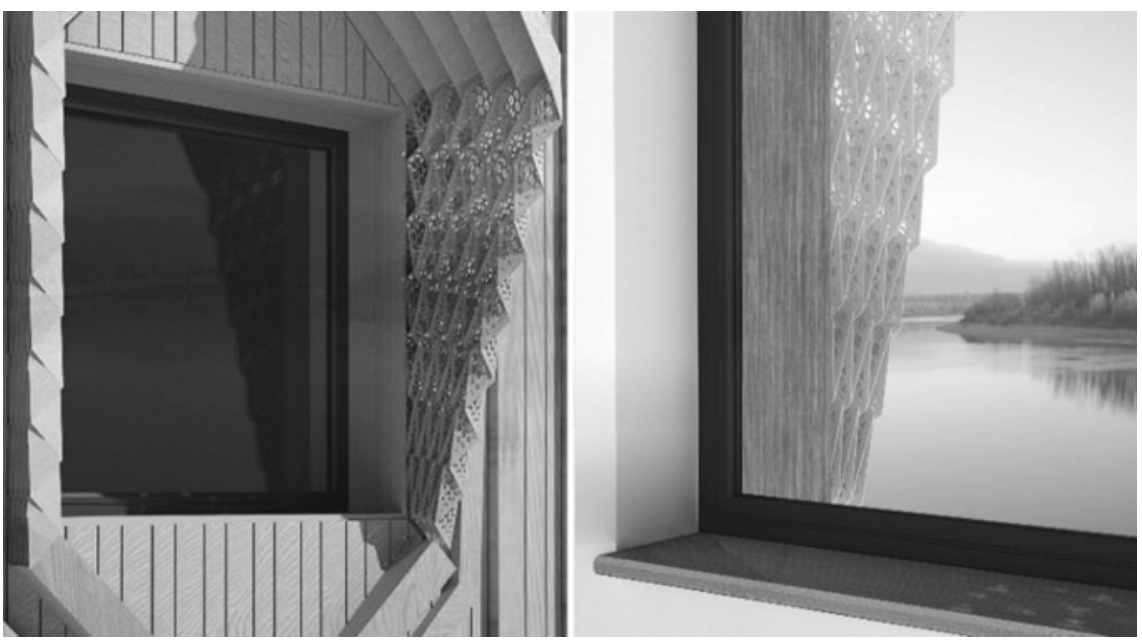

Fig. 3 Renders of exterior and interior views of the module

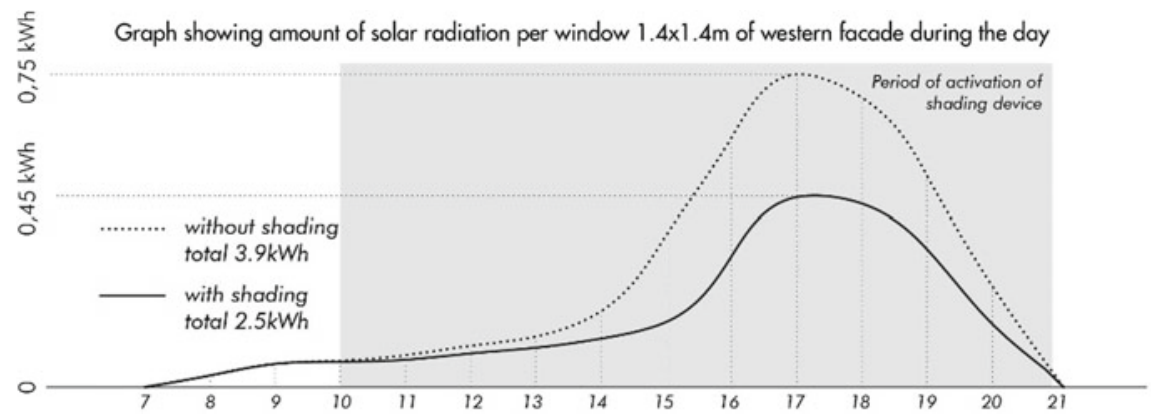

Fig. 4 Levels of solar radiation hitting the glass surface with and without the shading system

The results are then summed up to get the total amount of radiation per window. The simulation demonstrates that dynamic shading results in $35 \%$ reduction in radiation hitting the surface of the window (Fig. 4).

\section{Soft-Robotic Weather-Responsive Envelope}

The aim of the project is to harness the possibilities of the soft-robotic actuation into the development of a flexible and lightweight, adaptive building envelope. The innovative weather-responsive space for studying is equipped with a soft-robotic envelope system that transforms it radically from an open to enclosed thermally 
insulated space. Thus, it improves the climatic and energy performance. The design includes a bottom-up iterative process with use of simulations and optimization processes that focuses on the development of the soft robotic components.

Traditionally, all theories and techniques for robotic control, fabrication and sensing are based on a conventional definition of robots as a kinetic structure of rigid members. Recent advances in soft and smart materials, compliant mechanisms and nonlinear modeling have increasingly led to the popularization of the soft materials in robotics. This trend is driven by new scientific paradigms like biomimetics and morphological computation and by application requirements in biomedical engineering, service, rescue robots and other fields. The expectation is to make soft robots interact more easily and effectively with real-world environments (Mazzolai et al. 2012; Pfeifer et al. 2012).

The core concept of the soft robotics' is to fabricate a robot all made up of flexible and elastic components with the ability to change gaits easily and maneuver in very limited spaces, based on the use of shape changing materials and their composites (Yokoi et al. 1999). A variety of typologies of soft actuators were developed to this day, using different actuation mechanisms. Among these some of the more prominent are the PneuNets bending actuator, fiber-reinforced actuators, pneumatic artificial muscle, dielectric elastomer actuator and multi-module manipulator (De Falco et al. 2014).

\subsection{Methodology and Design Strategy}

The functional requirements for the design are derived from the need for temporary study and working space in institutions. Existing efforts usually suffer from compromised comfort. In this context, the requirements foresee the design of an outdoor study space designed to be easily assembled, disassembled and transported. It is to provide cover, sufficient illumination and ventilation and to be integrated with HVAC system and an insulating weather-responsive envelope to optimize energy efficiency.

With the soft robotics as a starting point, the design process takes a bottom-up strategy. It first focuses on the development of the soft envelope component and only then oversees in an iterative process the development of the overall design. The overall morphology of the envelope consists of eight identical faces of an octagonal dome configuration. Each of the envelope components requires two kind of motion: furl up to open and down to close (Fig. 5). This is possible by tweaking the inner structure with the pneumatic networks soft actuator (PneuNets).

The solution adapted to this project is a typology of PneuNets actuator that is designed specifically to create flat, compact soft robots that could squeeze through narrow spaces. These actuators use the PneuNets mechanism of work pressurizing a series of adjacent internal chambers. However, all chambers are embedded in a flat configuration inside the outlines of the body (Shepherd et al. 2011). Moreover, they use a variation in elastomer thickness to achieve their furling deformation. Attaching two embedded pneumatic networks back to back, we receive a compact bidirectional 


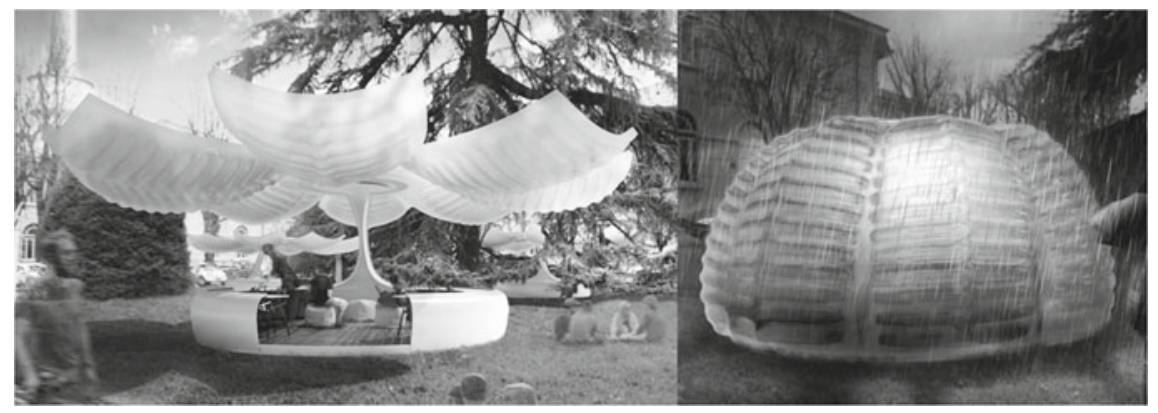

Fig. 5 Exterior renders of the shelter in open and closed configurations
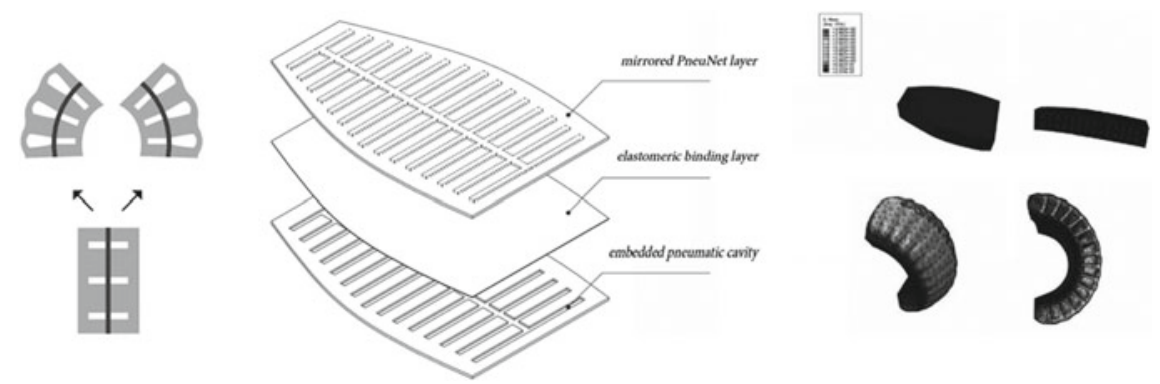

Fig. 6 Left to right: a diagram section of a flat bidirectional PneuNet actuator, when deflated and pressurized on each side; exploded axonometry of the soft-robotic skin component layers; FEM modelling and strain analysis for the soft component in full and sectioned view

actuator (Fig. 6). The result is a flat skin component that is able to adjust up or down, with a simple configuration, and only two air inlets to control the enclosure of the space.

The geometry is then optimized by design iterations, testing and adjusting through physical prototyping or digital simulations, using computational tools. This shows how the prototype will react under the different variables as pressure, gravity, wind and other loads; its deformation and trajectory. Once obtaining a satisfactory component design, the attention could be drawn to a broader scale of the skin system and its supporting structure.

\subsection{Simulation and Prototyping}

Designing a soft actuator, it is important to understand how the materials, morphology and geometry of the actuator do affect the behavior of the device. Dealing with elastic materials, it is difficult to accurately predict the effects of our design decisions due to their nonlinear behavior. Traditionally the design would be tested in a slow and 
costly process of physical prototyping. An increasingly more accessible approach is that of digital simulations using computational tools. These tools are used during the design phase in order to validate the design hypothesis and optimize it according to needs.

The first step with digital simulations is modeling the component in a $3 \mathrm{~d}$ modeling software. Some widely accessible tools familiar to designers for simulating the behavior of simple tensile and pneumatic structures are parametric design plugins such as Grasshopper for Rhinoceros and Kangaroo. This real-time physical simulation tool suffers some serious drawbacks. It allows getting some fast and basic sense of geometry and behavior and to simulate simple inflation. However, it lacks some important features in order to successfully replace physical prototyping, such as the ability to assign properties of physically accurate materials or different thicknesses.

Less accessible but more accurate tool is the Finite Element Method (FEM) using Abaqus or similar. FEM analysis is more adequate for the design of elastomeric PneuNet actuators for its physical accuracy. The process generally starts with the 3D digital model of the actuator, simplified, divided and imported into the FEM software. Materials are then created inserting values of physical properties and assigned to the model. The parts are then positioned correctly relative to one another and merged. Physical loads are defined and applied on the relevant parts of the model, as well as boundary conditions (e.g., anchoring or self-interaction) in order to obtain a realistic behavior. The final step is meshing the geometry and running the simulation (Fig. 6). Although very accurate, FEM is limited due to its complexity of set-up and long processing time, rendering impossible to change and take informed decisions in real-time.

Unlike small-scale soft actuators, large-scale actuators require the deployment of different solutions and technologies, especially for the fabrication of big-scale elastomer casting molds. One option of a low-cost fabrication is to cut and assemble elastomer mold parts using a CNC instead of 3d-printing it in one piece. This and some other methods are limited to simple geometries and might not be adapted for obtaining complex internal structures.

As a part of this project, a small-scale prototype was fabricated in order to test and compare the design for any discrepancies. The prototype was casted using two custom 3d-printed molds in three steps. Finally it was connected with tubes to an air pump controlled by an Arduino board, programmed to activate it in response to a light sensor input as a proof of concept (Fig. 7).

\section{Conclusion}

The pursuit for adaptive facade has led to a quest for intelligent control systems that integrate together many diverse functions. Nevertheless, the incorporation of these advanced systems in buildings is still slow due to high costs of materials and technologies and to the necessity of a deep research and development phase. 


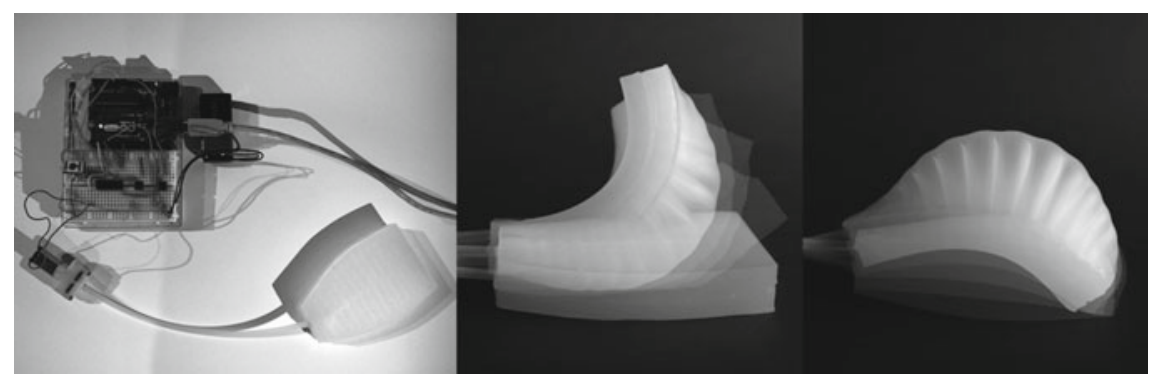

Fig. 7 Images of the scaled prototype with the Arduino control board configuration and actuation trajectory of the prototype in both directions

In fact, the two projects suggest that these systems need further investigations, accurate simulation software as well as a set of customized instructions for each application. On the other hand, they allow for a flexible design and endless possibilities of adaptation.

\section{References}

Addington M, Schodek DL (2005) Smart materials and new technologies: for the architecture and design professions. Architectural Press

Anishchenko M (2018) Smart memory materials in dynamic facade systems. Politecnico di Milano

De Falco I, Cianchetti M, Menciassi A (2014) A soft and controllable stiffness manipulator for minimally invasive surgery: preliminary characterization of the modular design. IEEE Eng Med Biol Soc

Huang WM, Ding Z, Wang CC et al (2010) Shape memory materials. Mater Today 13:54-61. https://doi.org/10.1016/S1369-7021(10)70128-0

Mazzolai B, Margheri L, Cianchetti M, Dario P, Laschi C (2012) Soft-robotic arm inspired by the octopus: from artificial requirements to innovative technological solutions, pp 338-339

Mok (2016) Wood-skin: composite material that's strong like wood, flexible like fabric. https:// www.treehugger.com/sustainable-productdesign/\%0Awood-skin-composite-wood-materialfolds-like-fabrics.html. Accessed 20 May 2019

Pfeifer R, Lungarella M, Iida F (2012) The challenges ahead for bio-inspired soft robotics. Commun ACM 55(11):76-87

Ritter A (2007) Smart materials in architecture, interior architecture and design. Birkhäuser

Schenk M, Guest SD (2014) On zero stiffness. J Mech Eng Sci 228:1701-1714. https://doi.org/10. $1177 / 0954406213511903$

Shepherd R, Ilievski F, Choi F, Morin S, Stokes A, Mazzeo A, Chen X, Wang M, Whitesides G (2011) Multigait soft robot. Proc Natl Acad Sci USA 108(51):20400-20403

Yokoi H, Yu W, Hakura J (1999) Morpho-functional machine: design of an amoebae model based on the vibrating potential method. Robot Auton Syst 28:217-236 
Open Access This chapter is licensed under the terms of the Creative Commons Attribution 4.0 International License (http://creativecommons.org/licenses/by/4.0/), which permits use, sharing, adaptation, distribution and reproduction in any medium or format, as long as you give appropriate credit to the original author(s) and the source, provide a link to the Creative Commons license and indicate if changes were made.

The images or other third party material in this chapter are included in the chapter's Creative Commons license, unless indicated otherwise in a credit line to the material. If material is not included in the chapter's Creative Commons license and your intended use is not permitted by statutory regulation or exceeds the permitted use, you will need to obtain permission directly from the copyright holder.

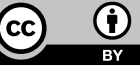

